\title{
Lee, Cadogan, and Chamberlain: an excellent point . . . But what about that iceberg?
}

\author{
Edward E. Rigdon
}

Received: 7 December 2012 / Accepted: 5 January 2013 / Published online: 16 January 2013

(C) Academy of Marketing Science 2013

It is an honor to be invited to comment on Nick, John and Laura's paper. The "formative measurement" theme has roiled methods literatures across the social sciences. Despite the number of publications on this topic, uncertainty remains widespread, and Lee et al.'s (2013) paper should resolve some of this. I agree with their argument that a MIMIC model does not represent simultaneous "formative measurement" and "reflective measurement." Factor-based structural equation modeling is, indeed, a factor-based method. If the MIMIC model is correct in the population, then the variance explained by the set of predictors, plus the variance of an error term if present, will exactly equal the variance of the factor which the MIMIC model engenders. Adding another predictor will not change this total variance -but using a different set of factor indicators may well change the total variance, demonstrating that the model is about the factor.

Still, I have significant differences with Lee, Cadogan and Chamberlain's perspective and recommendations. In my opinion, vague language lies at the root of the problem, so my own prescription would begin with scrapping the terms "construct," "latent variable," "reflective measurement," and "formative measurement" at least as they are currently used. Their recommendation regarding fixing weights is a fine idea, but our failure to fix model parameters more generally points to the failure of decades of published research to actually reveal much about the topics of study. Instead of the narrow issue and closely tailored solution presented by Lee, Cadogan and Chamberlain, researchers need to confront the fundamental, unresolved problems which lie at the very root of psychological measurement.

E. E. Rigdon $(\bowtie)$

Marketing Department, Georgia State University, P.O. Box 3991, Atlanta, GA 30302-3991, USA

e-mail: erigdon@gsu.edu

\section{Language}

In the course of their discussion, Lee, Cadogan and Chamberlain note, "The situation is not helped by the plethora of different terms used in the literature..." Rather than being only an aggravating circumstance, however, vagary of language is one of the most central problems not only in the "formative measurement" literature but across the field of psychological measurement. The terms "construct" (Maraun and Gabriel 2013; Rigdon 2012) and "latent variable" (as noted by Lee et al. 2013; Maraun 1996) both carry multiple meanings, with the result (and, one sometime suspects, the intent) that thinking is confused and distinctions are obscured.

In typical research situations, researchers working with multiple indicators operate at three levels of abstraction (Rigdon 2012). Researchers typically wish to make inferences at the most abstract level, about the behavior of variables which are inherently unobservable but whose existence is implied by theory. At the least abstract level, researchers make their inferences on the basis of data, collected by observation of one sort or another. At the intermediate level, in order to empirically evaluate their inferences, researchers form representations of the abstract variables using the observed variables. Depending on statistical method, these representations may be common factors, weighted composites, or something else.

"Construct" and "latent variable" are used to denote variables at both the most abstract level and at the intermediate level. In places, Lee, Cadogan and Chamberlain seem to use the term "latent variable" in precisely this way, referring to both conceptual variables and intermediate level representations. This blurring of distinctions has encouraged researchers to believe that their empirical representations, at the intermediate level, are equivalent to the conceptual variables standing at the highest level of abstraction (Maraun and Gabriel 2013). 
Despite the warnings of Cliff (1983) and others about the "nominalist fallacy" - thinking that something is identical with whatever label has been applied to it-researchers in the factor-analytic tradition have been particularly inclined to assume that the common factors derived in their statistical models are equivalent to the conceptual variable labels assigned to them.

Researchers should use language that clearly identifies the relevant level of abstraction. At the highest level of abstraction, researchers can refer to "conceptual variables." Conceptual variables are inherently unobservable and thus cannot themselves appear in statistical models which manipulate observations or functions of observations. At the lowest level of abstraction, researchers have "observed variables." At the intermediate level, instead of "constructs" or "latent variables," Rigdon (2012) recommended the term "proxy" to describe something standing in the place of a conceptual variable. The partial least squares path modeling literature has long used the term in precisely this way, although even in that literature, research reports usually refer to "the correlation between conceptual variables A and B" when they really mean "the correlation between the composite proxies for A and B." Lee, Cadogan and Chamberlain flirt with this very idea when they ask whether a "latent variable is a proxy for a 'real' entity"-although it is not entirely clear what "latent variable" means in their phrase.

Recognizing the factors and composites in statistical models as mere proxies for the conceptual variables of interest has a number of implications. Most significantly, this recognition implies that the crucial measurement question is not the connection between observed variables and a proxy but rather the connection between a proxy and a conceptual variable. The linkage between observed variables and a statistical proxy is purely a matter of mathematical operations. Researchers make inferences about the behavior of conceptual variables on the basis of the proxies, but the validity of these inferences depends on whether each proxy is a faithful representation of the conceptual variable. Nearly all currently popular tools for gauging measurement validity - tools like Average Variance Extracted or AVE (Fornell and Larcker 1981) focus on the connection between observed variables and proxy, while there is virtually nothing in the way of accepted tools and procedures for assessing the validity of proxies as stand-ins for conceptual variables. We might as well drop "formative measurement" and "reflective measurement" in favor of "composite proxy" and "factor proxy," and we ought to reserve questions of measurement and measurement validity for the relationship between concept and proxy.

Recognizing three levels of abstraction also has implications for the "causality" argument in measurement. Proxies are not in position to have causal relations with observed variables. If there is a causal story at this level, it involves a researcher who makes choices about how to build proxies out of observed variables. The question of causal relations, like the question of measurement itself, centers on the link between conceptual variable and proxy. Here, mere temporal ordering rules out treating the proxy, whether a factor proxy or a composite proxy, as the causal variable. A realist perspective insists that the conceptual variable transcends anything researchers build from data. A proxy comes into existence because a researcher is driven to create it in order to facilitate research about the conceptual variable, and the proxy is built using observed variables specifically chosen to yield a proxy to stand in the place of the conceptual variable. The conceptual variable can only be understood as cause, while the proxy must stand as an effect, though the causal process may be complex.

\section{Fixed weights vs. free weights}

Lee, Cadogan and Chamberlain criticize the practice of estimating "formative measurement" weights - the weights used to form composite proxies - as free parameters. Aside from the difficulties in freely estimating composite weights within a factor-based statistical method, Lee, Cadogan and Chamberlain echo claims elsewhere in the literature that this amounts to letting the data "define the construct." If by "construct," Lee, Cadogan and Chamberlain mean "conceptual variable," then their claim is unfounded because conceptual variables exist beyond the reach of data, consistent with the realist stance which they endorse. If by "construct" these authors mean "proxy," then they are correct that different weights produce different proxies, which may be more or less valid as stand-ins for a particular conceptual variable.

But it is hard to see how the argument applies only to composite proxies and not to factor proxies, particularly with factors themselves being (partly) weighted sums of observed variables. This may surprise some readers who remember being taught that the fundamental difference between factors and composites, such as those in principal components analysis, rests on this very point: composites are weighted functions of their indicators, while factors are not (Jöreskog 1979). Yet, the under-appreciated literature on factor indeterminacy (e.g., Steiger 1979) has long made clear that the difference is more subtle. Restating Lee, Cadogan and Chamberlain's Eq. 1 in matrix terms, define a model with a single factor proxy as:

$X=\Lambda \xi+\Delta$

where $\mathrm{X}$ is a set of observed variables, $\xi$ is the common factor proxy, and $\Delta$ is a vector of residuals, orthogonal with 
the factor proxy and among themselves. For simplicity, let the analysis be in population terms so that random sampling error is absent and assume that the factor model is perfectly consistent with the data at the population level. Standardize the factor proxy to a variance of 1 for the sake of identification. These conditions lead to a covariance matrix $\Sigma$ for the observed variables (since the model is correct at the population level, $\Sigma$ is both the empirical covariance matrix and the model-implied covariance matrix) of the form:

$\Sigma=\Lambda \Lambda^{\prime}+\Theta$

where the apostrophe indicates transposition and $\Theta$ is the covariance matrix of the residuals $\Delta$. Now, adapting Schönemann and Wang (1972, Eq. 2.10, p. 65) to the case of a model with one factor proxy, define a quantity $\mathrm{p}$ such that:

$p^{2}=1-\Lambda^{\prime} \Sigma^{-1} \Lambda$

The factor proxy $\xi$ can now be represented as the weighted sum:

$\xi=\Lambda^{\prime} \Sigma^{-1} X+p S$

Thus, the factor proxy is partly a weighted sum of the observed variables, weighted by their covariances and by their loadings. The other component, $\mathrm{S}$, is a random variable with unit variance. S must be orthogonal with the observed variables but is otherwise not constrained (Schönemann and Wang 1972, p. 65). S might be white noise, or might vary systematically with any variable excluded from the factor model (Schönemann and Steiger 1978). S could be any one of many, many random variables; thus, the factor $\xi$ is not one variable but rather an infinite variety of variables, depending on the choice of S. This is what is meant by factor indeterminacy. Researchers seek to minimize factor indeterminacy through specifying factor models with many observed variables per factor and strong loadings. To the extent that researchers succeed, the factor proxy becomes more purely a weighted sum of observed variables. If free weights are bad for composite proxies, then free loadings should be bad for factor proxies in almost precisely the same way.

Lee, Cadogan and Chamberlain call for composite proxy weights to be fixed on the basis of theory or prior knowledge. Could factor proxy loadings be fixed a priori on the same basis? Specifying models with parameters mostly fixedminimizing the number of free parameters - enhances rigor (Mulaik 2009, pp. 342-345) and increases statistical power (MacCallum et al. 1996, p. 142). After decades of experience in measuring psychological variables, surely we know the true values of many of the parameters in our models, at least for a large subset of the most prominent conceptual variables in major domains. For decades, researchers in the social sciences have been assessing the quality of their measures under the aegis of "construct validity" (Cronbach and Meehl 1955), a framework that relies upon knowledge of laws or law-like relationships linking the conceptual variables under study. The notion of construct validity assumes the existence of a nomologic network linking conceptual variables, just as laws in the physical sciences link matter and energy. Moreover, the development of valid measures should lead to further expansion of the network, linking an ever broader array of conceptual variables.

Even a cursory review of the applied literature will show that, after decades of research practice, researchers almost never specify their models with the preponderance of nonzero parameters such as factor loadings or regression weights fixed a priori based on prior knowledge. The problem for psychological measurement, and the problem for Lee, Cadogan and Chamberlain's recommendation, is that the psychological laws that would enable this approach are still not in hand (Borsboom et al. 2009). Indeed, the notion of construct validity was "born dead" (Borsboom et al. 2009 , p. 137). Decades of pretending that this fundamental knowledge was within our grasp have left our discipline painfully ignorant about its basic concepts, and the evidence of our ignorance is our insistence upon free parameters. John von Neumann is said to have remarked, "With four parameters I can fit an elephant, and with five I can make him wiggle his trunk" (Dyson 2004, p. 297). We will know that we know something when we see researchers optimizing fit by fixing parameters a priori (to conserve degrees of freedom), rather than optimizing fit by leaving parameters free. Even the broader adoption of Bayesian approaches (Kaplan and Paoli 2012; Scheines et al. 1999), which allow researchers to give explicit voice to prior knowledge, might be a sign that more researchers believe they actually know something about their field of study.

One phenomenon which applies to composite proxies but not factor proxies is collinearity. To the extent that a set of observed variables are predictors of the same dependent variable and are correlated, collinearity can lead to surprising regression weights. Positive zero-order correlations between predictors and dependent variable can become negative regression weights. These can strike researchers as unacceptable and contrary to theory, and Lee, Cadogan and Chamberlain's recommendation to use fixed regression weights would certainly prevent this. However, there are a number of other alternatives, besides fixing the weights, for solving this particular problem. One alternative is to use "correlation weights" (Waller and Jones 2010) instead of standard regression weights. Correlation weights ignore correlation among the predictors - the weight for each predictor is simply proportional to its correlation with the dependent variable. Correlation weights offer potential advantages over regression weights (Dana and Dawes 2004). Ordinary least 
squares regression weights, which take account of redundancy among predictors, offer optimal prediction in the sense of producing the highest $\mathrm{R}^{2}$ within the data used to estimate model parameters, when assumptions hold. Correlation weights, however, may yield superior out-ofsample prediction-prediction with data not used to estimate the weights - under certain common conditions (Dana and Dawes 2004). Such weights are available to researchers using partial least squares path analysis, where they are commonly known as Mode A outer weights (Dijkstra 2010; Rigdon 2013). Alternatives like correlation weights ought to be made available in more statistical packages.

Then again, simple unit or equal weights will perform fairly well under certain conditions (Einhorn and Hogarth 1975; Rozeboom 1979), and even purely random regression weights sometimes perform not much worse than optimal ordinary least squares weights (Wainer 1976). Depending on conditions, mandating fixed weights might not always induce substantial deterioration in performance, but such results may be more indicative of the nature of linear models than of a correct motivating theory.

\section{Factor analysis vs. measurement}

Composite proxies cannot be used in factor-based structural equation modeling, precisely because factor-based structural equation modeling is a factor-based method. Similarly, factor proxies cannot be used in partial least squares path modeling because that is a composite-based method. Lee, Cadogan and Chamberlain are certainly correct that MIMIC models, estimated via factor-based structural equation modeling, do not simultaneously instantiate a factor proxy and a composite proxy for the same conceptual variable because factor-based structural equation modeling does not enable composite proxies. The "interpretational confounding" noted by Lee, Cadogan and Chamberlain and others is easily dispelled; in a factor model, factors rule.

Despite the availability of composite-based methods - including partial least squares path modeling as well as others less widely used (Rigdon 2012) - researchers have rather stubbornly clung to the notion of incorporating composite proxies within factor-based structural equation modeling (Treiblmaier et al. 2011 report yet another attempt). As Lee, Cadogan and Chamberlain note, a pro-factor bias permeates the broader literature on psychological measurement, almost as if psychological measurement by definition were a factorbased activity. This bias is understandable, given that factor analysis and the classical true-score theory of measurement are fraternal twins, having both been introduced by Charles Spearman in papers published in the same year. Classical true score theory is simply a special case of the common factor model (McDonald 1999). Since the introduction of the twin innovations, it has been hard to separate psychological measurement from factor analysis. Some critiques of "formative measurement" (e.g., Edwards 2011) read as though the core objection is simply that the composite proxies of "formative measurement" are not factors - true enough, but not damning unless one believes that measurement necessitates common factors.

Researchers should welcome steps to combat this powerful bias. I would have appreciated more emphasis in Lee, Cadogan and Chamberlain's fine paper on non-factor solutions. Their recommendation compromises the composite side of the MIMIC structure but preserves the factor side. There may be better solutions which preserve the composite side while disposing of the factor side altogether (Schönemann and Steiger 1976). In their own way, Lee, Cadogan and Chamberlain themselves may have succumbed to factor obsession.

\section{Psychological measurement in crisis}

For a long time I have found the "formative measurement" literature frustratingly picayune, torturing marginal issues and contesting debate points with little real progress, nurturing raging controversies about what seems like mere mathematics. Meanwhile, it has been increasingly apparent that the practice of psychological measurement faces genuine and fundamental challenges. Critics argue persuasively that the practice of psychological measurement is built upon false premises and has broadly failed to yield insight. Decades of practice have left us still estimating models with free parameters, as if we enter each new study with minimal prior knowledge. Researchers equate the factor proxies in their models with conceptual variables on the strength of almost no evidence. Choosing to recognize the distinction between concept and proxy will only underline the difficulty that researchers face. Instead of touting measurement metrics like Average Variance Extracted, which are computed from factor model parameter estimates, researchers must finally confront the core question: how do you really assess the validity/faithfulness/verisimilitude of something built from data as a stand-in for something that is intrinsically unobservable?

At the most fundamental level, Michell (2009) argues that researchers in most domains have yet to establish the necessary conditions for measurement to occur. For a given instrument to be a measure, the instrument must measure something and that something must be measurable. To be measurable, the something must have quantitative structure, which is to say that it must have more than ordinal scale. If the something is only categorical, whether or not the categories are ordered, then the entity cannot be measured, though it can be assessed. Assessment is the task facing 
the physician attempting to diagnose a patient's condition. It is an important task in its own right, but it is not measurement. Commenting about his own instrument, intelligence testing pioneer Alfred Binet remarked, "The scale properly speaking does not permit the measure of the intelligence, because intellectual qualities are not superposable, and therefore cannot be measured as linear surfaces are measured, but are on the contrary a classification" (quoted in Michell 2012, p. 3). Within the domain of marketing, think about customer satisfaction. Does this variable really follow a quantitative continuum so that there is such a thing as being a little more satisfied or a little less? Reichheld's (2006) Net Promoter Score survey methodology divides customers into only three categories: Promoters (evangelists), Passives (the merely satisfied) and Detractors (the dissatisfied). Leaving aside controversy over Reichheld's method, many practicing marketers will find this typology of customers familiar. Pretending that factors and conceptual variables are identical mooted this question because common factors are quantitative by the nature of their construction and so that quality must apply to the equivalent conceptual variables. Overcoming this false equating of concept and factor (Maraun and Gabriel 2013), however, raises the question anew. If there are only categories, then measurement is formally impossible (Michell 2009).

The assumption that psychological variables are indeed measurable may have been adopted in willful disregard of the lack of supporting evidence. Quantitative measurement is a precondition for a discipline to be regarded as a science and to draw financial support from bodies that fund scientific research. Carefully ignoring the profitable yet dubious assumption of quantitative structure while carrying on with "research as usual" makes the practice of psychometrics "pathological" (Michell 2008). Instead of worrying the frayed remains of "formative measurement," researcher time would be better invested in understanding the broader domain of assessment and the implications of the distinction between measurement and assessment for the field of marketing.

Lee, Cadogan and Chamberlain suggest that elements of Rossiter's (2002, 2010) alternative "C-OAR-SE” approach are somewhat akin to their recommendations for establishing a priori values for composite weights. Rossiter's approach rejects the received view of measurement but also rejects the notion that it is possible to evaluate measurement validity in quantitative terms. Instead of quantifying validity, measures are judged by the researcher (or by a small committee of researchers) as being either highly valid or not valid. Aside from the immediate danger of confirmation bias (Nickerson 1998) and the seeming return to the operationalism long ago rejected by the psychological measurement community, this approach looks like a concession that genuine scientific measurement of psychological attributes is beyond our abilities. It looks like a surrender.
I cannot endorse giving up, though I do not wish to minimize the challenge confronting us. The task is both immense and crucial, which is why we need all of the bright minds available working on the central problem and not distracted by marginal issues. Only in this way will we succeed in putting psychological measurement on a firm footing. If, instead, we continue rearranging deck chairs, in 40 more years we will find ourselves exactly where we are now, knowing almost nothing about the conceptual variables that figure in our models, and estimating the same parameters again and again.

Could it really be that bad? Could there really be decades of research with nothing to show for it? Back in the 1970s, earthquake researchers expected to be able to predict major earthquakes accurately within the course of a decade with the help of high-speed computers and structural models (Silver 2012). Tragically, accurate prediction of major earthquakes still eludes us (Silver 2012). By contrast, prediction of weather phenomena has become highly accurate. The problem for earthquake researchers, unlike meteorologists, is that the phenomena which they study are largely unobservable, being obscured by miles and miles of rock. Despite the development of high-speed computers and the collection of immense bodies of data, earthquake prediction has advanced little over the state of the art in the 1970s. We in the social sciences must learn to deal with our own unobservables, or we are likely to fare no better.

\section{References}

Borsboom, D., Cramer, A. O. J., Kievit, R. A., Scholten, A. Z., \& Franić, S. (2009). The end of construct validity. In R. W. Lissitz (Ed.), The concept of validity: Revisions, new directions, and applications. Charlotte, NC: Information Age.

Cliff, N. (1983). Some cautions concerning the application of causal modeling methods. Multivariate Behavioral Research, 18(1), $115-126$.

Cronbach, L. J., \& Meehl, P. E. (1955). Construct validity in psychological tests. Psychological Bulletin, 52(4), 281-302.

Dana, J., \& Dawes, R. M. (2004). The superiority of simple alternatives to regression for social science prediction. Journal of Educational and Behavioral Statistics, 29(3), 317-331.

Dijkstra, T. K. (2010). Latent variables and indices: Herman Wold's basic design and partial least squares. In V. Esposito Vinzi, W. W. Chin, J. Henseler, \& H. Wang (Eds.), Handbook of partial least squares: Concepts, methods and applications. Berlin: Springer.

Dyson, F. (2004). A meeting with Enrico Fermi. Nature, 427, 297.

Edwards, J. R. (2011). The fallacy of formative measurement. Organizational Research Methods, 14(2), 37-388.

Einhorn, H. J., \& Hogarth, R. M. (1975). Unit weighting schemes for decision making. Organizational Behavior and Human Performance, 13(2), 171-192.

Fornell, C., \& Larcker, D. F. (1981). Evaluating structural equation models with unobservable variables and measurement error. Journal of Marketing Research, 18(1), 39-50.

Jöreskog, K. G. (1979). Basic ideas of factor and component analysis. In K. G. Jöreskog \& D. Sörbom (Eds.), Advances in factor 
analysis and structural equation models. Cambridge, MA: Abt Books.

Kaplan, D., \& Paoli, S. (2012). Bayesian structural equation modeling. In R. H. Hoyle (Ed.), Handbook of structural equation modeling. New York: Guilford.

Lee, N., Cadogan, J.W., \& Chamberlain, L. (2013). The MIMIC model and formative variables: Problems and solutions. AMS Review.

MacCallum, R. C., Browne, M. W., \& Sugawara, H. M. (1996). Power analysis and determination of sample size in covariance structure modeling. Psychological Methods, 1(2), 130-149.

Maraun, M. D. (1996). Metaphor taken as math: indeterminacy in the factor analysis model. Multivariate Behavioral Research, 31(4), $517-538$.

Maraun, M. D., \& Gabriel, S. G. (2013). Illegitimate concept equating in the partial fusion of construct validation theory and latent variable modeling. New Ideas in Psychology, 31(1), 32-42.

McDonald, R. P. (1999). Test theory: A unified treatment. Mahwah, NJ: Lawrence Erlbaum Associates.

Michell, J. (2008). Is psychometrics pathological science? Measurement, 6(1-2), 7-24.

Michell, J. (2009). Invalidity in validity. In R. W. Lissitz (Ed.), The concept of validity: Revisions, new directions, and applications. Charlotte, NC: Information Age.

Michell, J. (2012). Alfred Binet and the concept of heterogeneous orders. Frontiers in Psychology, 3, 261. doi:10.3389/fpsyg.2012.00261.

Mulaik, S. A. (2009). Linear causal modeling with structural equations. Boca Raton, FL: CRC Press.

Nickerson, R. S. (1998). Confirmation bias: a ubiquitous phenomenon in many guises. Review of General Psychology, 2(2), 175-220.

Reichheld, F. (2006). The ultimate question: Driving good profits and true growth. Cambridge, MA: Harvard Business School.

Rigdon, E. E. (2012). Rethinking partial least squares path modeling: in praise of simple methods. Long Range Planning, 45(5-6), 341358.
Rigdon, E. E. (2013). Partial least squares path modeling. In G. R. Hancock \& R. D. Mueller (Eds.), Structural equation modeling: A second course (2nd ed.). Charlotte, NC: Information Age.

Rossiter, J. R. (2002). The C-OAR-SE procedure for scale development in marketing. International Journal of Research in Marketing, 19(4), 305-335.

Rossiter, J. R. (2010). Measurement for the social sciences: The COAR-SE method and why it must replace psychometrics. New York: Springer.

Rozeboom, W. W. (1979). Sensitivity of a linear composite of predictor items to differential item weighting. Psychometrika, 44(3), 289296.

Scheines, R., Hoijtink, H., \& Boomsma, A. (1999). Bayesian estimation and testing of structural equation models. Psychometrika, 64 (1), 37-52.

Schönemann, P. H., \& Steiger, J. H. (1976). Regression component analysis. British Journal of Mathematical and Statistical Psychology, 29(2), 175-189.

Schönemann, P. H., \& Steiger, J. H. (1978). On the validity of indeterminate factor scores. Bulletin of the Psychonomic Society, 12 (4), 287-290.

Schönemann, P. H., \& Wang, M.-M. (1972). Some new results on factor indeterminacy. Psychometrika, 37(1), 61-91.

Silver, N. (2012). The signal and the noise: Why so many predictions fail-but some don't. New York: Penguin.

Steiger, J. H. (1979). Factor indeterminacy in the 1930s and the 1970s: some interesting parallels. Psychometrika, 44(1), 157-167.

Treiblmaier, H., Bentler, P. M., \& Mair, P. (2011). Formative constructs implemented via common factors. Structural Equation Modeling, 18(1), 1-17.

Wainer, H. (1976). Estimating coefficients in linear models: It don't make no nevermind. Psychological Bulletin, 83(2), 213-217.

Waller, N. G., \& Jones, J. A. (2010). Correlation weights in multiple regression. Psychometrika, 75(1), 58-69. 